\section{OPEN ACCESS}

Edited by:

Glen Jickling,

University of Alberta, Canada

Reviewed by:

Joseph Kamtchum Tatuene,

University of Alberta, Canada

Beom Joon Kim,

Seoul National University Bundang

Hospital, South Korea

*Correspondence:

Yuesong Pan

yuesongpan@ncrcnd.org.cn

tThese authors have contributed equally to this work

Specialty section:

This article was submitted to

Stroke,

a section of the journal

Frontiers in Neurology

Received: 09 August 2019 Accepted: 23 September 2019 Published: 10 October 2019

Citation:

Liu H, Meng X, Liu C-F, Wang D, Zheng H, Li H, Liu L, Wang Y, Wang Y and Pan Y (2019) Higher Hemoglobin

A1c Level Is Associated With Poor

Outcome of Intracerebral

Hemorrhage. Front. Neurol. 10:1073.

doi: 10.3389/fneur.2019.01073

\title{
Higher Hemoglobin A1c Level Is Associated With Poor Outcome of Intracerebral Hemorrhage
}

\begin{abstract}
Huihui Liu ${ }^{1+}$, Xia Meng ${ }^{2,3+}$, Chun-Feng Liu ${ }^{1}$, David Wang ${ }^{4}$, Huaguang Zheng ${ }^{2,3}$, Hao $\mathrm{Li}^{2,3}$, Liping Liu ${ }^{2,3}$, Yilong Wang ${ }^{2,3}$, Yongjun Wang ${ }^{2,3}$ and Yuesong Pan ${ }^{2,3 *}$

${ }^{1}$ Department of Neurology and Suzhou Clinical Research Center of Neurological Disease, The Second Affiliated Hospital of Soochow University, Suzhou, China, ${ }^{2}$ Department of Neurology, Beijing Tiantan Hospital, Capital Medical University, Beijing, China, ${ }^{3}$ China National Clinical Research Center for Neurological Diseases, Beijing, China, ${ }^{4}$ Barrow Neurological Institute Dignity Health, St. Joseph's Hospital and Medical Center, Phoenix, AZ, United States
\end{abstract}

Background: Pre-morbid chronic hyperglycemia is associated with the poor outcome of ischemic stroke, but the association between chronic hyperglycemia, and the long-term outcome of acute intracerebral hemorrhage is still poor understood.

Methods: Data on patients with acute intracerebral hemorrhage in the ACROSS-China registry (Abnormal Glucose Regulation in Patients With Acute Stroke Across China) were reviewed. Elevated hemoglobin A1c ( $\mathrm{HbA1c}$ ) level on admission was indicative of chronic hyperglycemia. According to the clinical categories of $\mathrm{HbA1c}$, patients were divided into three groups. Multivariable logistic regression or Cox method was performed to analyze the association of $\mathrm{HbA1c}$ and the prognosis of patients with acute intracerebral hemorrhage (poor functional outcome [modified Rankin scale score 3-6] and mortality) at 1 year.

Results: A total of 416 patients were included in this study. Fifty-two (12.5\%) patients died and 130 (31.8\%) had poor functional outcome at 1-year follow-up. The higher levels of $\mathrm{HbA1c}(\geq 6.5 \%)$ was associated with a poor functional outcome (OR $2.35,95 \% \mathrm{Cl}$, 1.28-4.29) and increased mortality (OR 2.63, 95\% Cl 1.34-5.15), compared with the lowest category. When further stratified by diabetic or non-diabetic medical history, higher $\mathrm{HbA1c}(\geq 6.5 \%)$ still increased the risk of poor functional outcome (OR $3.42,95 \% \mathrm{Cl}$ 1.39-8.44) and mortality (OR 4.48, 95\% Cl 1.64-12.24) in patients with non-diabetic medical history. However, higher HbA1c didn't have the association with the increased risk of poor functional outcome (OR 1.06, 95\% Cl 0.37-3.03) and mortality (OR 1.20, 95\% Cl 0.39-3.72) in patients with diabetic medical history.

Conclusions: Higher HbA1c was associated with a higher risk of death and poor functional outcome 1 year after intracerebral hemorrhage, especially in patients without a diabetic history.

Keywords: intracerebral hemorrhage, chronic hyperglycemia, hemoglobin A1c, outcome, diabetes mellitus 


\section{INTRODUCTION}

Diabetes mellitus or hyperglycemia as an independent risk factor for stroke is well-established. A number of studies have demonstrated that either acute or chronic hyperglycemia on admission, is associated with poor prognosis of ischemic stroke, resulting in disability or mortality (1-4). However, evidence on the association of glycemic status and outcomes in patients with intracerebral hemorrhage (ICH) remains limited. Because the pathophysiology of ICH is different from that of ischemic stroke, the association between the blood glucose level, and the outcome of ICH might be different from that of ischemic stroke.

Several researchers have reported the effect of acute glycemic levels on clinical outcomes (5-8). Since acute glycemic status is potentially influenced by the stress response in the setting of an illness per se; hemoglobin A1c (HbA1c) level, an indicator of the chronic glycemic state, can more accurately reflect a long-term endogenous exposure to glucose than glucose levels on admission. However, only a few of studies explored the association between $\mathrm{HbAlc}$ and the outcome of acute ICH (9, 10). Data from Get with The Guidelines (GWTG)-Stroke registry showed that higher levels of HbAlc $(>8.0 \%)$ increased the risk of in-hospital death in patients with ICH without a diabetic history; while lower HbA1c $(<5.7 \%)$ increased the risk of death in patients with or without history of diabetes mellitus (9). However, this study only examined the in-hospital outcomes in ICH, but without a longer term of follow up after discharge.

Therefore, it is unclear whether baseline HbA1c is also associated with long-term outcomes in patients with $\mathrm{ICH}$ as well. Hence, we conducted this study to explore the relationship between admission $\mathrm{HbA1c}$ and the 1-year prognosis of ICH using a nationwide, multicenter, prospective stroke registry in China.

\section{METHODS}

\section{Study Participants}

Data were derived from the ACROSS-China (Abnormal Glucose Regulation in Patients with Acute Stroke across China) registry. The rationale and design of ACROSS-China have been described previously (11). In brief, ACROSS-China registry was a prospective, multicenter, cohort study that consecutively enrolled patients with acute cerebrovascular events, including ischemic stroke, ICH and subarachnoid hemorrhage (SAH) in 35 hospitals across China from 2008 to 2009. The purpose of ACROSSChina intended to investigate the association between abnormal glucose regulation and the outcome of patients after an acute stroke within 14 days from the event onset. The diagnosis of ICH was based on the World Health Organization criteria (12) and combined with brain CT or MRI findings. The study was approved by the ethics committee of Beijing Tiantan Hospital. Informed consent was obtained from all patients who were enrolled before the data collection.

\section{Data Collection}

Demographic, vascular risk factors and baseline clinical data were collected through face-to-face interviews by trained neurologists from participating hospitals within $24 \mathrm{~h}$ after admission. Vascular risk factors included a history of hypertension, diabetes mellitus, dyslipidemia, coronary heart disease, atrial fibrillation, heart failure, family history of stroke, tobacco, and alcohol use. Baseline clinical data included National Institutes of Health Stroke Scale (NIHSS), Glasgow coma scale (GCS) score, pre-morbid modified Rankin Scale (mRS) score, systolic blood pressure, and diastolic blood pressure, body mass index (BMI), hematoma location and laboratory values. Hematoma location was divided into lobes, basal ganglia region, brain stem, cerebellum, extension to surrounding spaces. Laboratory values on admission included fasting glucose, $\mathrm{HbAlc}$, high density lipoprotein, triglyceride.

$\mathrm{HbA1c}$ was measured within $24 \mathrm{~h}$ after admission by using the high performance liquid chromatographicanalysis (HPLC) and in alignment with the Diabetes Control and Complications Trial and National Glycohemoglobin Standardization Program (NGSP) standards (13). Patients were divided into three groups according to their HbA1c levels ( $<5.7,5.7-6.4$, $\geq 6.5 \%$ ) (1416). In addition, the in-hospital complications of pneumonia and urinary tract infection (UTI) were also included.

\section{Follow-Up and Outcome Assessment}

Patients were followed up for their functional outcome and all-cause mortality at 1 year after the onset of symptom. All follow-up was performed by the trained interviewers who used a standardized protocol through centralized telephone interviews. Poor functional outcome was defined as a score of 3-6 on the mRS (17). Death was confirmed either by the death certificate from the local citizen registry or hospital where the patient was treated.

\section{Statistical Analysis}

The baseline and clinical characteristics were compared between the groups. Continuous variables were presented as mean $\pm \mathrm{SD}$ or median [interquartile range [IQR]] and categorical variables were presented as frequency (\%). ANOVA or the KruskalWallis test was used for continuous variables and the $\chi^{2}$ test for categorical variables.

The univariable and multivariable-adjusted odds ratios (ORs) with $95 \%$ confidence intervals (CIs) for poor functional outcome were calculated by using a logistic regression model and the univariable and multivariable-adjusted hazard ratios (HRs) with 95\% CIs for death were calculated by using a Cox regression model. The lowest clinical categories of HbAlc was used as the reference. Age, sex, and other significant covariates in the univariable analysis were adjusted in the multivariable model. The cumulative probability of death according to the HbA1c categories were assessed by Kaplan-Meier curves and were compared by the log-rank test.

All analyses were conducted with SAS version 9.4 software (SAS Institute Inc., Cary, NC). Two-tailed $p<0.05$ were considered to be statistically significant.

\section{RESULTS}

A total of 649 patients with ICH were enrolled in this prospective stroke registry. After excluding 233 patients due to missing HbA1c data and lost to follow up, 416 were included in the final 
analysis (Table 1). Baseline and clinical features of the included and excluded patients were well-balanced except that the HbA1c level of the included participants was higher than those excluded $(p=0.002)$.

Among included patients, the average age was $58.8 \pm 13.2$ years and $64.9 \%$ of them were male. The median HbA1c was $5.7 \%$ (IQR, 5.2-6.3\%). Baseline features of the patients according to the clinical categories of HbAlc were showed in Table 2. Patients with a higher HbAlc levels were more likely to have history of diabetes mellitus, dyslipidemia, higher fasting glucose and triglycerides levels, and be on hypoglycemic therapy. In addition, NIHSS and GCS scores were imbalanced between the groups.

\section{Association of HbA1c and Clinical Outcomes}

During a 1-year follow-up, a total of $52(12.5 \%)$ patients died and $130(31.8 \%)$ had poor functional outcome. Cumulative probability of death was shown in Figure 1. The association of HbAlc and the clinical outcomes of patients with ICH was demonstrated in Table 3.

Compared with the lowest category of HbAlc, the highest category of $\mathrm{HbAlc}$ was associated with the poor functional outcome (OR: $1.67,95 \% \mathrm{CI}: 1.0-2.80, p=0.05$ ) and increased all-cause mortality (HR: 1.95, 95\% CI: 1.03-3.68, $p=0.04$ ) (Table 3). After adjustment for age, sex, medical history of dyslipidemia, NIHSS score, GCS score, and triglyceride, the highest category of HbAlc was still associated with the poor functional outcome (adjusted OR: 2.35, 95\% CI: 1.28-4.29, $p=$ 0.006 ) and increased all-cause mortality (adjusted HR: $2.63,95 \%$ CI: $1.34-5.15, p=0.005)$.

When stratified by a positive or negative medical history of diabetes mellitus, the highest clinical categories of HbAlc in patients without history of diabetes mellitus was associated with both poor functional outcome (adjusted OR: 3.42, 95\% CI: 1.39$8.44, p=0.008$ ) and increased all-cause mortality (adjusted HR: 4.48, 95\% CI: $1.64-12.24, p=0.004$ ) (Table 4). However, we did not find any association between higher clinical categories of $\mathrm{HbAlc}$ and poor outcomes in $\mathrm{ICH}$ patients with a history of diabetes mellitus.

\section{DISCUSSION}

This multicenter cohort study demonstrated that chronic hyperglycemia prior to the onset of $\mathrm{ICH}$ was associated with poor functional outcome and increased mortality. This association was significant as HbA1c levels increased. However, when further stratified by medical history with or without diabetes mellitus, we found that chronic hyperglycemia increased the risk of a worsened clinical outcome after the onset of $\mathrm{ICH}$, especially in patients without a diabetic history.

Diabetes mellitus is an established independent risk factor for stroke. The prevalence of previously diagnosed diabetes mellitus in stroke patients was estimated between 10 and $20 \%$, while undiagnosed diabetes mellitus and impaired glucose tolerance accounted for an additional 5-28\% (18). A number of studies showed that admission hyperglycemia was a common
TABLE 1 | The characteristics of the excluded and included patients.

\begin{tabular}{|c|c|c|c|}
\hline & $\begin{array}{l}\text { Excluded } \\
(n=233)\end{array}$ & $\begin{array}{l}\text { Included } \\
(n=416)\end{array}$ & $p$-value \\
\hline Age (year), mean (SD) & $58.9 \pm 12.7$ & $58.8 \pm 13.2$ & 0.90 \\
\hline Sex (male), $n(\%)$ & $146(62.7)$ & 268 (64. 9) & 0.57 \\
\hline Tobacco use, $n(\%)$ & & & 0.74 \\
\hline Never & $149(64.0)$ & 255 (61.3) & \\
\hline Quit & $18(7.7)$ & $38(9.1)$ & \\
\hline Current & 66 (28.3) & $123(29.6)$ & \\
\hline $\begin{array}{l}\text { Body mass index, }\left(\mathrm{kg} / \mathrm{m}^{2}\right) \text {, } \\
\text { mean }(S D)\end{array}$ & $24.9 \pm 4.7$ & $24.5 \pm 3.8$ & 0.36 \\
\hline \multicolumn{4}{|l|}{ Medical history, $\boldsymbol{n}$ (\%) } \\
\hline Diabetes mellitus & $20(8.6)$ & $38(9.1)$ & 0.81 \\
\hline Hypertension & $146(62.7)$ & $284(68.3)$ & 0.15 \\
\hline Dyslipidemia & $19(8.2)$ & $18(4.3)$ & 0.04 \\
\hline Coronary heart disease & $18(7.7)$ & $33(7.9)$ & 0.93 \\
\hline Atrial fibrillation & $3(1.3)$ & $6(1.4)$ & 1.00 \\
\hline Heart failure & $0(0.0)$ & $1(0.3)$ & 1.00 \\
\hline Family history of stroke & $18(8.8)$ & $41(10.6)$ & 0.48 \\
\hline \multicolumn{4}{|l|}{ On admission status } \\
\hline NIHSS score, mean (SD) & $9.7 \pm 8.2$ & $8.7 \pm 7.6$ & 0.09 \\
\hline median (IQR) & $9.0(3.0-13.0)$ & $7.0(2.0-13.0)$ & \\
\hline GCS score, mean (SD) & $13.0 \pm 3.0$ & $13.3 \pm 2.6$ & 0.35 \\
\hline median (IQR) & $15.0(12.0-15.0)$ & $15.0(12.0-15.0)$ & \\
\hline Pre-morbid mRS & $6(2.6)$ & $5(1.2)$ & 0.33 \\
\hline \multicolumn{4}{|l|}{ score> =2, $n(\%)$} \\
\hline $\mathrm{SBP}(\mathrm{mmHg})$, mean $(\mathrm{S} D)$ & $155.2 \pm 24.1$ & $155.1 \pm 23.6$ & 0.80 \\
\hline DBP $(m m H g)$, mean $(S D)$ & $91.5 \pm 14.2$ & $90.7 \pm 14.1$ & 0.43 \\
\hline Hematoma location, $n(\%)$ & & & 0.95 \\
\hline Lobes & $26(11.2)$ & $52(12.5)$ & \\
\hline Basal ganglia region & $168(72.1)$ & $300(72.1)$ & \\
\hline Brain stem & $16(6.9)$ & $23(5.5)$ & \\
\hline Cerebellum & $13(5.6)$ & $22(5.3)$ & \\
\hline $\begin{array}{l}\text { Extension to surrounding } \\
\text { spaces }\end{array}$ & $10(4.3)$ & $19(4.6)$ & \\
\hline \multicolumn{4}{|c|}{ Laboratory values on admission } \\
\hline $\begin{array}{l}\text { Fasting glucose, } \\
\text { (mmol/L), mean (SD) }\end{array}$ & $6.3 \pm 2.4$ & $6.2 \pm 2.1$ & 0.84 \\
\hline HbA1c (\%), Mean $\pm S D$ & $5.5 \pm 1.1$ & $5.8 \pm 1.3$ & 0.002 \\
\hline Median (IQR) & $5.4(4.8-5.9)$ & $5.7(5.2-6.3)$ & \\
\hline $\begin{array}{l}\text { High density lipoprotein } \\
(\mathrm{mmol} / \mathrm{l}) \text {, mean }(S D)\end{array}$ & $1.4 \pm 0.7$ & $1.3 \pm 0.5$ & 0.33 \\
\hline $\begin{array}{l}\text { Triglyceride }(\mathrm{mmol} / \mathrm{L}) \\
\text { mean }(S D)\end{array}$ & $1.7 \pm 1.4$ & $1.8 \pm 1.4$ & 0.32 \\
\hline \multicolumn{4}{|l|}{ Medication in hospital, $n$ (\%) } \\
\hline Oral hypoglycemic agents & $20(8.6)$ & $38(9.1)$ & 0.81 \\
\hline Insulin administration & $19(8.2)$ & $31(7.5)$ & 0.75 \\
\hline Antihypertensive therapy & $151(64.8)$ & $284(68.3)$ & 0.37 \\
\hline \multicolumn{4}{|c|}{ In-hospital complications $n(\%)$} \\
\hline Pneumonia & $42(18.0)$ & $61(14.7)$ & 0.26 \\
\hline UTI & $11(4.7)$ & $31(7.5)$ & 0.18 \\
\hline
\end{tabular}

NIHSS, National Institutes of Health Stroke Scale; SD, standard deviation; IQR, interquartile; SAH, subarachnoid hemorrhage; Q, quartile; GCS, Glasgow coma scale; $S B P$, systolic blood pressure; DBP, diastolic blood pressure; $m R S$, modified Rankin scale; HDA1C, hemoglobin A1C; UTI, urine tract infection. 
TABLE 2 | Baseline characteristics according to HbA1c categories.

\begin{tabular}{|c|c|c|c|c|}
\hline Variable & $\begin{array}{c}\text { HbA1c }<5.7 \% \\
(n=194)\end{array}$ & $\begin{array}{l}\text { HbA1c 5.7-6.4\% } \\
(n=129)\end{array}$ & $\begin{array}{c}\mathrm{HbA} 1 \mathrm{c} \geq 6.5 \% \\
(n=93)\end{array}$ & $p$-Value \\
\hline Age (year), mean (SD) & $57.5 \pm 14.1$ & $59.5 \pm 12.9$ & $60.3 \pm 11.3$ & 0.15 \\
\hline Male, $n(\%)$ & $121(63.0)$ & $90(70.3)$ & $57(61.3)$ & 0.29 \\
\hline Tobacco use, $n$ (\%) & & & & 0.53 \\
\hline Never & $126(64.9)$ & $74(57.4)$ & $55(59.1)$ & \\
\hline Quit & $16(8.2)$ & $11(8.5)$ & $11(11.8)$ & \\
\hline Current & $52(26.8)$ & $44(34.1)$ & $27(29.0)$ & \\
\hline Alcohol intake, $n(\%)$ & $37(19.1)$ & $26(20.2)$ & $12(12.9)$ & 0.33 \\
\hline Body mass index, $\left(\mathrm{kg} / \mathrm{m}^{2}\right)$ & $24.1 \pm 3.4$ & $24.8 \pm 4.2$ & $24.9 \pm 3.9$ & 0.24 \\
\hline \multicolumn{5}{|l|}{ Medical history, $n$ (\%) } \\
\hline Diabetes mellitus & $4(2.1)$ & $7(5.4)$ & 27 (29.0) & $<0.001$ \\
\hline Hypertension & $122(62.9)$ & $93(72.1)$ & $69(74.2)$ & 0.08 \\
\hline Dyslipidemia & $3(1.5)$ & $7(5.4)$ & $8(8.6)$ & 0.02 \\
\hline Coronary heart disease & $12(6.2)$ & $12(9.3)$ & $9(9.7)$ & 0.47 \\
\hline Atrial fibrillation & $3(1.5)$ & $2(1.6)$ & $1(1.1)$ & 0.94 \\
\hline Heart failure & $1(0.5)$ & $0(0.0)$ & $0(0.0)$ & 0.57 \\
\hline Family history of stroke & $15(8.2)$ & $12(9.8)$ & $14(17.1)$ & 0.09 \\
\hline \multicolumn{5}{|l|}{ On admission status } \\
\hline NIHSS score, median (IQR) & $8(4-15)$ & $5(2-11)$ & $6(2-11)$ & $<0.001$ \\
\hline GCS score, median (IQR) & $14.0(11.0-15.0)$ & $15.0(13.0-15.0)$ & $15.0(13.5-15.0)$ & $<0.001$ \\
\hline $\mathrm{SBP}(\mathrm{mmHg})$, mean $(S D)$ & $155.5 \pm 23.0$ & $153.2 \pm 24.2$ & $157.0 \pm 24.2$ & 0.43 \\
\hline DBP (mmHg), mean (SD) & $90.9 \pm 14.4$ & $89.8 \pm 13.7$ & $91.4 \pm 13.9$ & 0.96 \\
\hline Hematoma location, $n$ (\%) & & & & 0.71 \\
\hline Lobes & $24(12.4)$ & $19(14.7)$ & $9(9.7)$ & \\
\hline Basal ganglia region & $142(73.2)$ & $91(70.5)$ & $67(72.0)$ & \\
\hline Brain stem & $13(6.7)$ & $4(3.1)$ & $6(6.5)$ & \\
\hline Cerebellum & $9(4.6)$ & $7(5.4)$ & $6(6.5)$ & \\
\hline Extension to surrounding spaces & $6(3.1)$ & $8(6.2)$ & $5(5.4)$ & \\
\hline \multicolumn{5}{|l|}{ Laboratory values on admission } \\
\hline Fasting glucose, (mmol/L), mean (SD) & $5.6 \pm 1.2$ & $5.7 \pm 1.3$ & $7.9 \pm 3.2$ & $<0.001$ \\
\hline High density lipoprotein (mmol/l), mean (SD) & $1.33 \pm 0.35$ & $1.32 \pm 0.35$ & $1.34 \pm 0.74$ & 0.25 \\
\hline Triglyceride (mmol/L), mean (SD) & $1.60 \pm 1.45$ & $1.83 \pm 1.22$ & $1.94 \pm 1.33$ & 0.02 \\
\hline \multicolumn{5}{|l|}{ Medication in hospital, $\boldsymbol{n}(\%)$} \\
\hline Oral hypoglycemic agents & $4(2.1)$ & $6(4.7)$ & $28(30.1)$ & $<0.001$ \\
\hline Insulin administration & $8(4.1)$ & $5(3.9)$ & $18(19.4)$ & $<0.001$ \\
\hline Antihypertensive therapy & $135(69.6)$ & $81(62.8)$ & $68(73.1)$ & 0.23 \\
\hline Diuretics & $17(8.8)$ & $10(7.8)$ & $8(8.6)$ & 0.95 \\
\hline Beta blockers & $14(7.2)$ & $7(5.4)$ & $5(5.4)$ & 0.75 \\
\hline \multicolumn{5}{|l|}{ In-hospital complications, $\boldsymbol{n}(\%)$} \\
\hline Pneumonia & $32(16.5)$ & $18(14.0)$ & $11(11.8)$ & 0.56 \\
\hline UTI & $16(8.2)$ & $7(5.4)$ & $8(8.6)$ & 0.57 \\
\hline
\end{tabular}

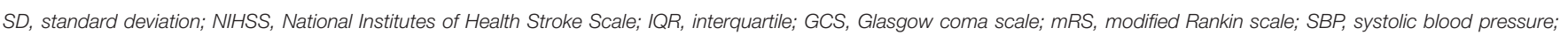
DBP, diastolic blood pressure; SAH, subarachnoid hemorrhage; HbA1C, hemoglobin A1c; UTI, urine tract infection.

manifestation in the acute stage of stroke, and always correlated with a poor prognosis and mortality $(6,7,19)$. A post hoc study of INTERACT2 (Intensive Blood Pressure Reduction in Acute Cerebral Hemorrhage Trial) showed that baseline hyperglycemia was related to 90 -days poor clinical outcome following acute ICH (6). A single-center study showed admission hyperglycemia was associated with the increased risk of in-hospital mortality in ICH patients (7). Unlike the blood glucose level on admission, which is influenced by acute stress response, $\mathrm{HbA1c}$ represents an extended-period of glycemic level and is a far more reliable and stable test than admission blood glucose level to detect the newly diagnosed diabetes mellitus or the glycemic status in known diabetes mellitus prior to the onset of stroke. Only two cohort studies explored the association between $\mathrm{HbAlc}$ and outcome of $\mathrm{ICH}$ and found that baseline $\mathrm{HbAlc}$ was related with poor outcome $(9,10)$. However, these two studies only examined inhospital outcome, but without long-term follow up. Our study added evidence that higher HbAlc was associated with 1-year poor functional outcome and increased mortality. Our finding also indicated that diabetes mellitus itself, rather than the level of $\mathrm{HbA1c}$, predicts poor outcome or death in patients with ICH. Therefore, prevention of diabetes mellitus rather than acute glycemic control is more useful in preventing poor outcome, a finding similar to the results of The Stroke Hyperglycemia 
Insulin Network Effort (SHINE) trial (20). In addition, patients with known diabetes and normal values of $\mathrm{HbA1c}$ had a similar outcome with those with known diabetes and higher values of

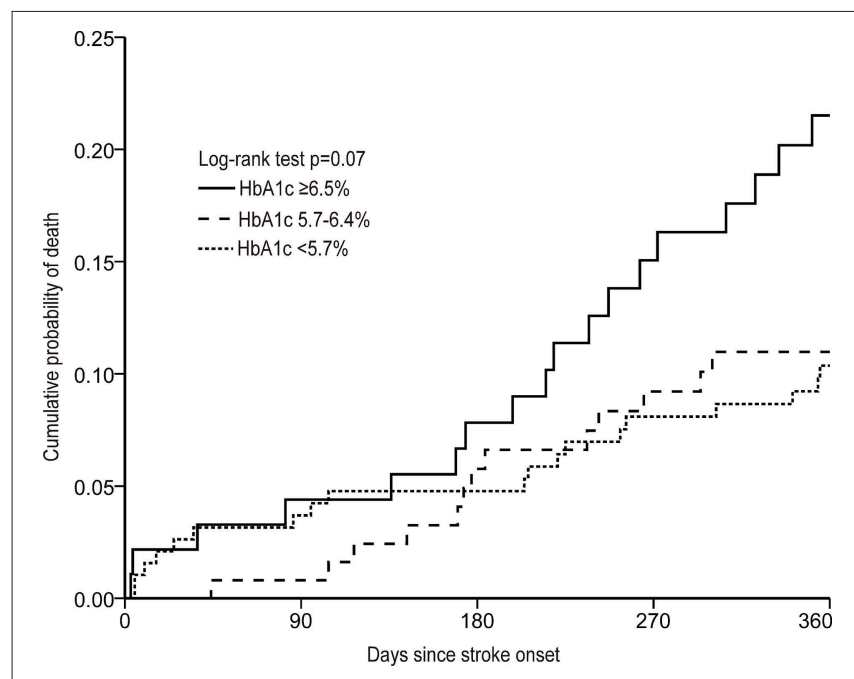

FIGURE 1 | Cumulative probability of death according to $\mathrm{HbA1c}$ categories at baseline in patients with intracerebral hemorrhage.
HbA1c. This phenomenon might be confounded by potential covariants, such as the duration of diabetic state and pre-morbid mRS, which was missing in ACROSS-China study.

$\mathrm{HbA1c}$ level reflects the average blood glucose status between 80 and 120 days. The elevated HbAlc is indicative of poor control of hyperglycemia for a period of time. The effect of hyperglycemia on patients with ICH may be related to the exacerbation of hematoma expansion and perihematoma edema $(21,22)$. This effect may result from the disturbance of osmotic-hemostasis state (23). In animal models, hyperglycemia can cause more profound brain edema and increase neuronal death in areas around the hematoma (24). However, in this study, the data on the volumes of hematoma and perihematoma edema was missing, except that the hematoma location was available, which appeared similar in sizes between groups.

There are several limitations to our study. First, the sample size was a little small. A large-scale study is needed to validate this association. Second, 233 patients were excluded due to missing HbA1c level or no follow ups. Nevertheless, the baseline features between the included patients and those excluded were well-balanced. Third, selection bias might be present because most of patients included in this registry had mild to moderate ICH. Fourth, the population in our study were of Chinese descent; these results may not be generalizable to patients of other ethnicities.

TABLE 3 | Association between clinical categories of HbA1c and poor outcomes of intracerebral hemorrhage.

\begin{tabular}{|c|c|c|c|c|c|c|c|}
\hline Outcomes & HbA1c levels & $N$ & Events, $n(\%)$ & \multicolumn{2}{|c|}{ Unadjusted } & \multicolumn{2}{|c|}{ Adjusted } \\
\hline \multirow[t]{3}{*}{${ }^{\ddagger}$ Poor functional outcome } & $<5.7 \%$ & 193 & 59 (30.6) & Ref. & - & Ref. & - \\
\hline & $5.7-6.4 \%$ & 124 & $32(25.8)$ & $0.79(0.48-1.31)$ & 0.36 & $1.11(0.62-2.00)$ & 0.73 \\
\hline & $\geq 6.5 \%$ & 92 & $39(42.4)$ & $1.67(1.0-2.80)$ & 0.05 & $2.35(1.28-4.29)$ & 0.006 \\
\hline Death & $<5.7 \%$ & 194 & 20 (10.3) & Ref. & - & Ref. & - \\
\hline
\end{tabular}

OR, Odds ratio; HR, hazard ratio; Cl; confidence interval; HbA1c, hemoglobin A1c and Q, quartile.

*HR for death, while OR for poor functional outcome.

${ }^{\dagger}$ Adjusted for age, sex, medical history dyslipidemia, NIHSS score, GCS score, triglyceride.

¥Poor functional outcome: modified Rankin Scale score of 3-6.

TABLE 4 | Association between clinical categories of HbA1c levels and poor outcomes of intracerebral hemorrhage stratified by diabetic or non-diabetic medical history at 1-year.

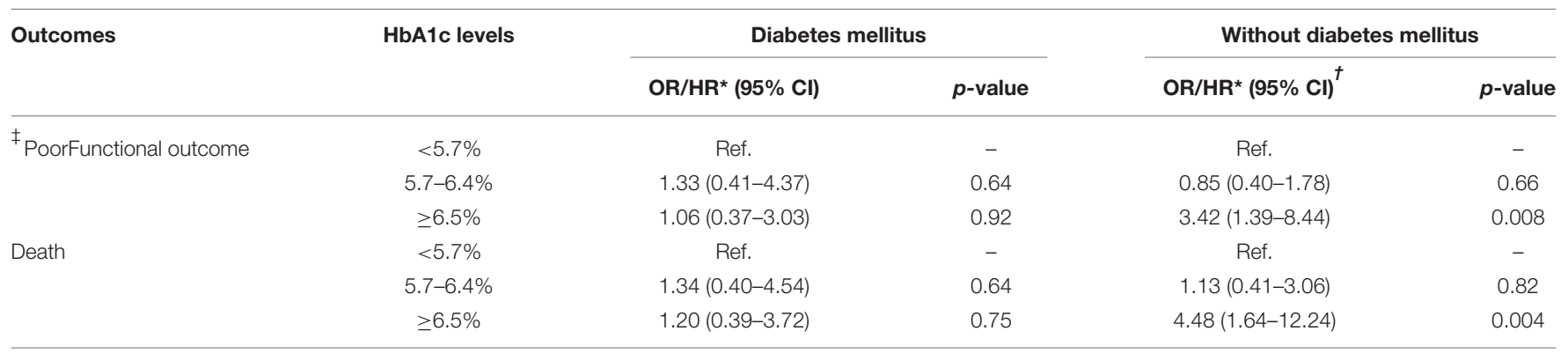

OR, Odds ratio; HR, hazard ratio; Cl; confidence interval; HbA1c, hemoglobin A1c and Q, quartile.

*HR for death, while OR for poor functional outcome.

${ }^{\dagger}$ Adjusted for age, sex, medical history of dyslipidemia, GCS score, NIHSS score, triglyceride.

¥Poor functional outcome: modified Rankin Scale score of 3-6. 


\section{CONCLUSIONS}

Our study demonstrated that higher levels of HbAlc at baseline were associated with increased risks of death and poor functional outcome 1 year after $\mathrm{ICH}$, especially significant in patients without a diabetic history.

\section{DATA AVAILABILITY STATEMENT}

The datasets generated for this study will not be made publicly available. Data are available to researchers on request for purposes of reproducing the results or replicating the procedure by directly contacting the corresponding author.

\section{ETHICS STATEMENT}

The studies involving human participants were reviewed and approved by the ethics committee of Beijing Tiantan Hospital. The patients/participants provided their written informed consent to participate in this study.

\section{REFERENCES}

1. Luitse MJ, Velthuis BK, Kappelle LJ, van der Graaf Y, Biessels GJ, Group DS. Chronic hyperglycemia is related to poor functional outcome after acute ischemic stroke. Int J Stroke. (2017) 12:180-6. doi: 10.1177/17474930166 76619

2. Wu S, Wang C, Jia Q, Liu G, Hoff $\mathrm{K}$, Wang X, et al. HbAlc is associated with increased all-cause mortality in the first year after acute ischemic stroke. Neurol Res. (2014) 36:444-52. doi: 10.1179/1743132814Y.00000 00355

3. Masrur S, Cox M, Bhatt DL, Smith EE, Ellrodt G, Fonarow GC, et al. Association of acute and chronic hyperglycemia with acute ischemic stroke outcomes post-thrombolysis: findings from get with the guidelines-stroke. $J$ Am Heart Assoc. (2015) 4:e002193. doi: 10.1161/JAHA.115.002193

4. Stead LG, Gilmore RM, Bellolio MF, Mishra S, Bhagra A, Vaidyanathan L, et al. Hyperglycemia as an independent predictor of worse outcome in nondiabetic patients presenting with acute ischemic stroke. Neurocrit Care. (2009) 10:181-6. doi: 10.1007/s12028-008-9080-0

5. Koga M, Yamagami H, Okuda S, Okada Y, Kimura K, Shiokawa Y, et al. Blood glucose levels during the initial $72 \mathrm{~h}$ and 3 -month functional outcomes in acute intracerebral hemorrhage: the SAMURAI-ICH study. J Neurol Sci. (2015) 350:75-8. doi: 10.1016/j.jns.2015.02.018

6. Saxena A, Anderson CS, Wang X, Sato S, Arima H, Chan E, et al. Prognostic significance of hyperglycemia in acute intracerebral hemorrhage: the INTERACT2 study. Stroke. (2016) 47:682-8. doi: 10.1161/STROKEAHA.115.011627

7. Snarska KK, Bachorzewska-Gajewska H, Kapica-Topczewska K, Drozdowski W, Chorazy M, Kulakowska A, et al. Hyperglycemia and diabetes have different impacts on outcome of ischemic and hemorrhagic stroke. Arch Med Sci. (2017) 13:100-8. doi: 10.5114/aoms.2016.61009

8. Tapia-Perez JH, Gehring S, Zilke R, Schneider T. Effect of increased glucose levels on short-term outcome in hypertensive spontaneous intracerebral hemorrhage. Clin Neurol Neurosurg. (2014) 118:37-43. doi: 10.1016/j.clineuro.2013.12.018

9. Dandapat S, Siddiqui FM, Fonarow GC, Bhatt DL, Xu H, Matsouaka R, et al. A paradoxical relationship between hemoglobin A1C and in-hospital mortality in intracerebral hemorrhage patients. Heliyon. (2019) 5:e01659. doi: 10.1016/j.heliyon.2019.e01659

\section{AUTHOR CONTRIBUTIONS}

YP and HLiu concepted and designed the study. YP and HLi acquired data, analyzed, and interpreted data. HLiu and XM drafted and edited manuscript. C-FL and DW revised and edited the manuscript. YiW, LL, and $\mathrm{HZ}$ contributed and designed the study. YoW supported, supervised, and designed the study.

\section{FUNDING}

This work was supported by grants from the Ministry of Science and Technology of the People's Republic of China (2016YFC0901002, 2016YFC0901001, 2017YFC1310901, 2017YFC1310902, 2017YFC1307905, 2018YFC1311700, and 2018YFC1311706) and National Natural Science Foundation of China (81701149, 81600999, 81971091), grants from Beijing Municipal Administration of Hospitals (SML20150502, QML20190501), grants from Beijing Municipal Science \& Technology Commission (D171100003017002, D151100002015003), grants from National Science and Technology Major Project (2017ZX09304018), and Jiangsu Provincial Medical Key Discipline Project (ZDXKB 2016022).

10. Zhang G, Wu F, Xu Y, Feng J, Cai Z, Xu B, et al. Prestroke glycemic status is associated with the functional outcome in spontaneous intracerebral hemorrhage. Neurol Sci. (2015) 36:927-34. doi: 10.1007/s10072-014-2057-1

11. Jia Q, Zheng H, Zhao X, Wang C, Liu G, Wang Y, et al. Abnormal glucose regulation in patients with acute stroke across China: prevalence and baseline patient characteristics. Stroke. (2012) 43:650-7. doi: 10.1161/STROKEAHA.111.633784

12. Stroke-1989. Recommendations on stroke prevention, diagnosis, and therapy. Report of the WHO Task Force on Stroke and other Cerebrovascular Disorders. Stroke. (1989) 20:1407-31. doi: 10.1161/01.STR.20.10.1407

13. Pietrzak A, Chabros P, Grywalska E, Kicinski P, Pietrzak-Franciszkiewicz K, Krasowska D, et al. Serum lipid metabolism in psoriasis and psoriatic arthritis - an update. Arch Med Sci. (2019) 15:369-75. doi: 10.5114/aoms.2018.74021

14. American Diabetes A. Diagnosis and classification of diabetes mellitus. Diabetes Care. (2010) 33 (Suppl. 1):S62-9. doi: 10.2337/dc10-S062

15. World Health Organization. Use of Glycated Haemoglobin (HbAlc) in the Diagnosis of Diabetes Mellitus: Abbreviated Report of a WHO Consultation. Geneva: WHO Guidelines Approved by the Guidelines Review Committee (2011).

16. Selvin E, Rawlings AM, Bergenstal RM, Coresh J, Brancati FL. No racial differences in the association of glycated hemoglobin with kidney disease and cardiovascular outcomes. Diabetes Care. (2013) 36:2995-3001. doi: $10.2337 / \mathrm{dc} 12-2715$

17. Pan Y, Jing J, Chen W, Zheng H, Jia Q, Mi D, et al. Post-glucose load measures of insulin resistance and prognosis of nondiabetic patients with ischemic stroke. J Am Heart Assoc. (2017) 6:e004990. doi: 10.1161/JAHA.116.004990

18. van Kooten F, Hoogerbrugge N, Naarding P, Koudstaal PJ. Hyperglycemia in the acute phase of stroke is not caused by stress. Stroke. (1993) 24:1129-32. doi: 10.1161/01.STR.24.8.1129

19. Ntaios G, Egli M, Faouzi M, Michel P. J-shaped association between serum glucose and functional outcome in acute ischemic stroke. Stroke. (2010) 41:2366-70. doi: 10.1161/STROKEAHA.110.592170

20. Johnston KC, Bruno A, Pauls Q, Hall CE, Barrett KM, Barsan W, et al. Intensive vs. standard treatment of hyperglycemia and functional outcome in patients with acute ischemic stroke: the SHINE randomized clinical trialeffect of intensive vs. standard treatment of hyperglycemia on functional outcome in strokeeffect of intensive vs. standard treatment of hyperglycemia on functional outcome in stroke. JAMA. (2019) 322:326-35. doi: 10.1001/jama.2019.9346 
21. Chen R, Ovbiagele B, Feng W. Diabetes and stroke: epidemiology, pathophysiology, pharmaceuticals and outcomes. Am J Med Sci. (2016) 351:380-6. doi: 10.1016/j.amjms.2016. 01.011

22. Stead LG, Jain A, Bellolio MF, Odufuye A, Gilmore RM, Rabinstein A, et al. Emergency Department hyperglycemia as a predictor of early mortality and worse functional outcome after intracerebral hemorrhage. Neurocrit Care. (2010) 13:67-74. doi: 10.1007/s12028-0109355-0

23. Saltzman AJ, Stone GW, Claessen BE, Narula A, Leon-Reyes S, Weisz $\mathrm{G}$, et al. Long-term impact of chronic kidney disease in patients with ST-segment elevation myocardial infarction treated with primary percutaneous coronary intervention: the HORIZONS-AMI (Harmonizing Outcomes With Revascularization and Stents in Acute Myocardial Infarction) trial. JACC Cardiovasc Interv. (2011) 4:1011-9. doi: 10.1016/j.jcin.2011. 06.012
24. Song EC, Chu K, Jeong SW, Jung KH, Kim SH, Kim M, et al. Hyperglycemia exacerbates brain edema and perihematomal cell death after intracerebral hemorrhage. Stroke. (2003) 34:2215-20. doi: 10.1161/01.STR.0000088060.83709.2C

Conflict of Interest: The authors declare that the research was conducted in the absence of any commercial or financial relationships that could be construed as a potential conflict of interest.

Copyright (c) 2019 Liu, Meng, Liu, Wang, Zheng, Li, Liu, Wang, Wang and Pan. This is an open-access article distributed under the terms of the Creative Commons Attribution License (CC BY). The use, distribution or reproduction in other forums is permitted, provided the original author(s) and the copyright owner(s) are credited and that the original publication in this journal is cited, in accordance with accepted academic practice. No use, distribution or reproduction is permitted which does not comply with these terms. 